\title{
Changes in the expression of endothelial monocyte-activating polypeptide II in the rat hippocampus following status epilepticus
}

\author{
CHUN LI $^{1 *}$, WEINING MA ${ }^{2 *}$, YAJUAN ZHAO $^{1}$ and HUA WANG ${ }^{1}$ \\ ${ }^{1}$ Department of Pediatrics, Shengjing Hospital of China Medical University, Shenyang, Liaoning 110004; \\ ${ }^{2}$ Department of Neurosurgery, Shengjing Hospital of China Medical University, Shenyang, Liaoning 110022, P.R. China
}

Received May 25, 2020; Accepted November 10, 2020

DOI: $10.3892 /$ ijmm.2020.4808

\begin{abstract}
Endothelial monocyte-activating polypeptide II (EMAP II) is a sensitive marker of neurotoxic injury, the expression of which increases significantly under conditions of stress, such as hypoxia or apoptosis. Studies have confirmed the extensive apoptosis of nerve cells in the brain following status epilepticus (SE), and the occurrence of SE can confer a hypoxic state on cells. The purpose of the present study was to observe the changes in the expression of EMAP II, and in the numbers and tight junction protein levels of microvascular endothelial cells in the hippocampus of rats with pilocarpine-induced SE. The protein expression levels of EMAP II, CD31, zonula occludens 1 (ZO-1) and occludin in the hippocampus were determined by immunofluorescence and western blot analyses. It was found that almost $75.6 \%$ of the rats in the SE group developed Racine stage IV-V seizures at approximately $44.7 \pm 18.8 \mathrm{~min}$ after the pilocarpine administration, and the 24 -h mortality rate was almost $10.4 \%$. The weight of the rats in the SE group was significantly decreased within $24 \mathrm{~h}$ following SE. Immunofluorescence staining revealed a low EMAP II expression in the hippocampus of the rats in the control group; however, the numbers of EMAP II-positive cells were significantly increased in the SE group from $2 \mathrm{~h}$ to 21 days. The trend of EMAP II protein expression was consistent with that obtained with immunofluorescence staining. The numbers of CD31-positive microvascular endothelial cells were significantly increased from $24 \mathrm{~h}$ to 21 days compared with the levels in the control group. The protein expression of ZO-1 and occludin was most significantly decreased in the SE group. On the whole, the present study demonstrated that the expression of EMAP II in
\end{abstract}

Correspondence to: Professor Hua Wang, Department of Pediatrics, Shengjing Hospital of China Medical University, 36 Sanhao Street, Shenyang, Liaoning 110004, P.R. China

E-mail:wangh1_medicine@163.com

${ }^{*}$ Contributed equally

Key words: endothelial monocyte-activating polypeptide II, status epilepticus, brain microvascular endothelial cells, CD31 the rat hippocampus was upregulated in the SE model, which may promote angiogenesis and alter the TJ integrity of brain microvascular endothelial cells, with an increased number of CD31-positive microvascular endothelial cells and a decreased expression of ZO-1 and occludin.

\section{Introduction}

Epilepsy is one of the most common clinical neurological diseases, with a high incidence. The prevalence of epilepsy worldwide is approximately $6.38-7.60 \%(1,2)$. The pathogenesis of epilepsy is not very clear, although studies have confirmed that the normal function of brain cells requires a stable neurovascular unit, suggesting that vascular endothelial dysfunction may be related to its pathogenesis $(3,4)$.

Endothelial monocyte-activating polypeptide II (EMAP II), which is derived from its precursor aminoacyl-tRNA synthetase-interacting multi-functional protein 1 (AIMP1), has a molecular weight of approximately $22 \mathrm{kDa}$. It can promote cell apoptosis, inhibit tumor angiogenesis and mediate inflammation (5). EMAP II has a wide range of biological associations with endothelial cells. It can stimulate the expression of P-selectin and E-selectin, promote the release of von Willebrand factor (vWF) in endothelial cells, and induce the production of tissue factors on the surface of endothelial cells, activating pro-coagulant activity. In addition, it can promote the secretion of tumor necrosis factor (TNF)- $\alpha$ and interleukin (IL) -8 by monocytes, regulate the expression of TNF- $\alpha$ receptor 1 , and enhance the sensitivity of endothelial cells to TNF- $\alpha$-induced apoptosis $(5,6)$. The majority of research into EMAP II has focused on its roles in tumors, diabetes, atherosclerosis, chronic myocardial infarction and lung injury (7-10).

Studies have found that EMAP II is a sensitive marker of neurotoxic injury in a variety of central nervous system diseases, such as autoimmune encephalomyelitis and traumatic brain injury $(11,12)$. Mice with genetic defects in the EMAP II precursor, AIMP1, can develop degenerative diseases of the motor neuron axons or muscle atrophy and exhibit movement dysfunction (13). Patients with the homozygous deletion of the AIMP1 gene exhibit developmental delays, refractory epilepsy, or poor myelination of the brain (14). However, research into the relationship between EMAP II and epilepsy is rare.

AIMP1 is enzymatically converted into EMAP II under hypoxic conditions or in response to certain chemotherapeutic 
drugs or apoptosis (15-17). Knies et al (15), through in vitro experiments, confirmed that EMAP II can be detected in the cultures of apoptotic cells, but not in those of necrotic cells, suggesting that the enzymolysis and the release of EMAP II may be associated with apoptosis. Some researchers have found that caspase 3, which has an apoptosis-promoting function, can significantly increase EMAP II expression in the lungs, with caspase inhibitors reducing EMAP II expression (18). Barnett et al (16) found that the induction of apoptosis or the necrosis of prostate cancer cells allowed AIMP1 to be released into the extracellular space and cleaved to form EMAP II. These experiments suggest that apoptotic cells can produce EMAP II and that EMAP II can itself promote cell apoptosis.

The CD31 molecule is also known as platelet endothelial cell adhesion molecule-1 (PECAM-1). Intracranial CD31 is mainly found at the tight junction between endothelial cells, and its immunohistochemical detection can be used to prove the presence of endothelial cells and evaluate angiogenesis. Angiogenesis is often associated with the development of epilepsy. Clinically, compared with non-epileptic patients, the vascular density in the hippocampal tissue of patients with chronic refractory temporal lobe epilepsy is significantly increased, is positively associated with the frequency of epileptic seizures, and is unrelated to the etiology and degree of neuronal loss (19). In cultured hippocampal brain slices, following the epileptic discharge induced by kainic acid, the numbers of cells exhibiting laminin and rat endothelial cell antigen (RECA-1) immunofluorescence are significantly increased, the vascular density and branching are augmented, and the tight junction protein, zonula occludens 1 (ZO-1), is downregulated (20).

Neurons in the hippocampus have been reported to be hypoxic several seconds before, during, and after the seizure, particularly the pyramidal cell layer of the CA1 region (21). In addition, extensive neuronal apoptosis and pyroptosis have been identified to be involved in epileptogenesis (22). Therefore, it was hypothesized that the expression of EMAP II in the rat brain may become altered following status epilepticus (SE). Accordingly, the present study examined the changes in the expression of EMAP II and the tight junction proteins, ZO-1 and occluding, as well as in the numbers of CD31-positive microvascular endothelial cells in the rat hippocampus following SE.

\section{Materials and methods}

Animals and groups. A total of 240 healthy male Sprague-Dawley rats, 21 days old and weighing 45-65 g, were used in the present study. The rats were kept at a room temperature of $22-24^{\circ} \mathrm{C}$ and under a 12 a.m./12 p.m. light/dark cycle. All animal experiments were approved by the Ethics Committee of Shengjing Hospital Affiliated to China Medical University (no. 2016PS201K).

All the rats were randomly divided into 2 groups as follows: i) The control group $(n=120)$, which received an intraperitoneal injection of saline; and ii) the SE group $(n=120)$, which received an intraperitoneal injection of lithium chloride-pilocarpine. The time of SE appearance in the experimental group was considered $0 \mathrm{~h}$. In total of 6 time points, were selected for both groups: 2 and $24 \mathrm{~h}$, and 3, 7, 14 and 21 days. A total of 20 rats were selected at each time point.
Establishment of animal models of SE. According to the model described in the previous studies $(23,24)$, the rats in the model group were intraperitoneally injected with lithium chloride solution [3 mEq $/ \mathrm{kg}$ (127 mg/kg), Sigma-Aldrich; Merck KGaA]. Subsequently, 18-20 h later, the rats in the model group were intraperitoneally injected with pilocarpine solution $(30 \mathrm{mg} / \mathrm{kg}$, Sigma-Aldrich; Merck KGaA); at 30 min prior to the pilocarpine administration, the rats were intraperitoneally injected with bromide scopolamine solution $(1 \mathrm{mg} / \mathrm{kg}$, Sigma-Aldrich; Merck KGaA) to antagonize the peripheral cholinergic reaction induced by pilocarpine. Level IV-V Racine seizures, according to the rat grade evaluation standard (25), were considered to indicate a successful model. If no seizures occurred after the first dose of pilocarpine, an additional injection of pilocarpine (10 $\mathrm{mg} / \mathrm{kg}$ dose) was administered every $30 \mathrm{~min}$ until the rats developed level IV-V Racine seizure attacks. The maximum dose of pilocarpine intraperitoneally administered to each rat was not $>60 \mathrm{mg} / \mathrm{kg}$. The rats in the control group were administered the same amount of normal saline via intraperitoneal injection. To reduce the mortality rate of the epileptic rats, diazepam solution $(10 \mathrm{mg} / \mathrm{kg})$ was intraperitoneally injected into the rats after the SE lasted 30 min to terminate the SE attack as described in a previous study (24). The weights of the rats in each group were measured before their drug treatment and sampling. Samples from the 2 groups were collected at 6 time points after modeling. Rats were gas-anesthetized by inhaling 5\% isoflurane mixed with $50 / 50 \%$ oxygen/nitrogen. When the limbs were fixed and the tail pinched, there was no significant limb response, the rats were subjected to abdominal aorta bleeding until cardiac arrest, followed by sampling. At different time points following SE, the intact brain tissues from 6 rats were obtained and fixed in 4\% paraformaldehyde for morphological analysis, while hippocampal tissues from another 6 rats were collected and stored in a freezer at $-80^{\circ} \mathrm{C}$ for biological analysis.

Electroencephalogram recording of rats with SE. At 3 days before modeling, all the rats were administered $5 \%$ isoflurane mixed with 50/50\% oxygen/nitrogen as an inducible anesthesia, and the isoflurane concentration was subsequently reduced to $2 \%$ to maintain the anesthesia. Their heads were fixed in the stereoscopic locator in the prone position to fully expose the anterior fontanelle. The cross-point of the anterior fontanelle of the rats was selected as the zero point with reference to the 5th edition of Paxinos \& Watson brain stereolocation map (26), and then 2 points $4-\mathrm{mm}$ posterior and 2-mm lateral to the anterior fontanelle were selected. The electrode needles were inserted to a depth of approximately $4 \mathrm{~mm}$ under the skull and were fixed by tissue glue. The rats were placed in a shield and the electroencephalogram (EEG) was used before and after model establishment.

Immunofluorescence staining. Brain tissues fixed in $4 \%$ paraformaldehyde were embedded in paraffin and then cut into 4- $\mu$ m-thick sections. Brain sections were dewaxed with xylene, dehydrated with a gradient of alcohol, and then subjected to a microwave antigen retrieval method (for EMAP II, the sections were heated with $0.01 \mathrm{~mol} / 1$ sodium citrate buffer, $\mathrm{pH} 6.0$, and for CD31 they were heated with Tris-EDTA buffer, $\mathrm{pH}$ 9.0). The sections were incubated with normal goat serum to reduce 
non-specific staining and then with primary antibody at $4{ }^{\circ} \mathrm{C}$ overnight. The primary antibodies comprised rabbit anti-rat EMAP II polyclonal antibody (1:100, 11091-1-AP, ProteinTech Group, Inc.) and mouse anti-rat CD31 monoclonal antibody (1:200, ab64543, Abcam). According to the primary antibody source, goat anti-rabbit polyclonal secondary antibody (1:200, ab150077, Abcam) or goat anti-mouse polyclonal secondary antibody (1:200, ab150115, Abcam) were added the following day in a wet box in the dark at room temperature. DAPI (C1005, Biyuntian Institute of Biotechnology) was added for 5 min in the dark at room temperature. The sections were then observed under a microscope (Nikon Eclipse 80i; Nikon Corporation). The control group was incubated with phosphate-buffered saline (PBS) instead of primary antibodies. To quantify the EMAP II- and CD31-positive cells, 10 microscopic fields of the CA1, CA3 and dentate gyrus (DG) regions were randomly observed at x200 magnification. Image Pro Plus 6.0 image analysis software was used to determine the optical density value.

Western blot analysis. Hippocampal tissue was incubated in RIPA lysis buffer (PMSF, 100:1; P0013B, ST505, Biyuntian Institute of Biotechnology), homogenized under ultrasound and then pyrolysed at $4^{\circ} \mathrm{C}$ for $30 \mathrm{~min}$. The tissue was then centrifuged at $4^{\circ} \mathrm{C}$ for $15 \mathrm{~min}$ at $18,800 \mathrm{x}$ g. The protein concentration of the supernatant was determined with the BCA protein assay kit (P0010S, Biyuntian Institute of Biotechnology). The supernatant was mixed with $5 \mathrm{X}$ buffer and denatured in a water bath at $100^{\circ} \mathrm{C}$. Sample proteins in each subgroup were adjusted to the same volume and concentration according to the measured protein concentration. Proteins $(40 \mu \mathrm{g})$ were then separated using various concentrations (10\% for EMAP II; $6 \%$ for ZO-1 and occludin) of Bis-Tris sodium dodecyl sulfate-polyacrylamide electrophoresis gel (SDS-PAGE; Beyotime Institute of Biotechnology), according to the molecular weight of the target protein. The separated proteins were then transferred to a PVDF membrane. The PVDF membrane was then placed in 5\% skim milk and blocked for $2 \mathrm{~h}$ at room temperature prior to incubation with primary antibodies at $4^{\circ} \mathrm{C}$ overnight. The primary antibodies used included mouse anti-rat EMAP II monoclonal antibody (1:1,000, ab15693, Abcam), rabbit anti-rat ZO-1 polyclonal antibody (1:500, WL03419, Biological Technology Co., Ltd.), rabbit anti-rat occludin polyclonal antibody (1:500, WL03419, Biological Technology Co., Ltd.) and rabbit anti-rat alpha-tubulin antibody (1:10,000,112-1-24 AP, ProteinTech Group, Inc.). The following day, the membrane was incubated for $2 \mathrm{~h}$ with goat anti-rabbit secondary antibody $(1: 7,000$, sa00001-2, ProteinTech Group, Inc.) or goat anti-mouse secondary antibody (1:7,000, sa00001-1, ProteinTech Group, Inc.). Protein bands were visualized using ECL (NCI5079; Thermo Fisher Scientific, Inc.) and imaged using an electrophoresis gel imaging system. ImageJ software (version 1.4.3.67) was used to analyze the gray values of the western blot results, and the gray values of the target proteins were normalized to that of $\alpha$-tubulin.

Statistical analysis. SPSS 22.0 statistical software was used to analyze the data. The data are presented as the means \pm standard deviation, and an independent sample t-test was used to compare the data between the 2 groups. $\mathrm{P}<0.05$ was considered to indicate a statistically significant difference.

\section{Results}

Behavioral changes in SE rats. Following the establishment of the model, peripheral cholinergic reactions, such as erect hair and salivation were observed after $13.4 \pm 2.1 \mathrm{~min}$ in the rats in the SE group administered pilocarpine. Level IV-V Racine seizures developed in $75.6 \%$ of rats in the SE group. After the convulsion lasted for $30 \mathrm{~min}$, diazepam solution was intraperitoneally injected to terminate the SE attack. Within $24 \mathrm{~h}$, the rats still had repeated convulsions, with an average of $5.6 \pm 1.2$ convulsions per hour. The 24 -h mortality rate of the rats was approximately $10.4 \%$.

Body weight changes in rats with SE. Prior to model establishment, no significant differences in body weight were observed between the rats in the control and SE groups. Following the administration of lithium chloride, no significant differences in body weight were observed between the 2 groups. Following the administration of pilocarpine, the rats in the control group gained weight regularly at 5-12 g per day. By contrast, the rats in the SE group exhibited significant weight loss $24 \mathrm{~h}$ after SE compared with the controls with a maximum of $27.0 \%$ body weight loss and then began to gain weight. At 3 days, their body weight returned to the pre-model weight, although it was still significantly lower than that of the control group. At 7 days, there was still a difference in body weight between the 2 groups. Subsequently, the weights of the rats in the SE group recovered, and no significant difference in the weights of the rats were observed between the 2 groups at 14 days (Fig. 1A).

EEG monitoring of rats with SE. Following model establishment, the EEG of control group rats revealed a basic rhythm of $\alpha$ and $\theta$ waves of a low-to-middle amplitude of approximately $50 \mu \mathrm{V}$. The EEG of the SE group revealed high-amplitude spike wave emission with a frequency of approximately 10-20 Hz (Fig. 1B-D).

Changes inmicrovascular endothelialcells in the hippocampus of rats with SE. Compared with the control group at the same time point, the number of endothelial cells with positive CD31 staining in the hippocampal CA1, CA3 and DG areas began to increase significantly from $24 \mathrm{~h}$ in the SE group, with the optical density being significantly higher in the SE group than in the control group $(\mathrm{P}<0.001)$. The number of CD31-positive endothelial cells peaked in the SE group at 3 days, and the optical density value was significantly higher in the SE group than in the control group $(\mathrm{P}<0.001)$. At 21 days, the number of CD31-positive endothelial cells was significantly higher in the SE group than in the control group at the same time point (Figs. 2 and $\mathrm{S} 1$ ).

Changes in the expression of EMAP II in the hippocampus of rats with SE. EMAP II was expressed at low levels in the hippocampal CA1, CA3 and DG areas of the control group. However, the numbers of EMAP II-positive cells were higher in the SE group at $2 \mathrm{~h}$. In addition, the optical density 


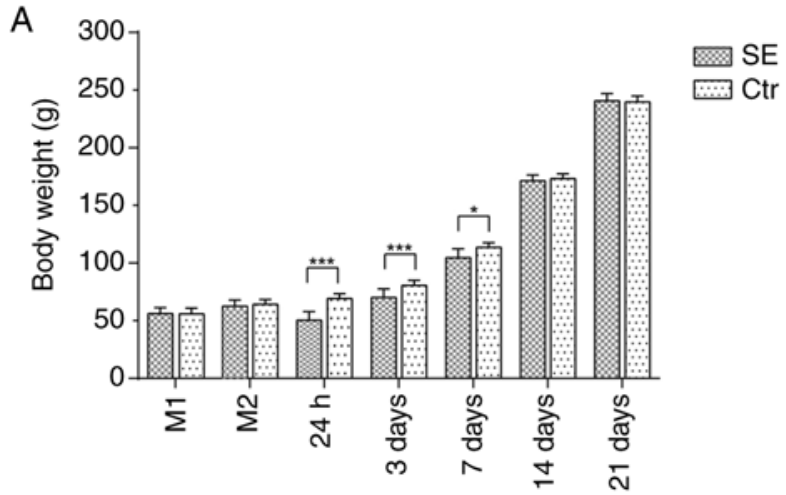

B

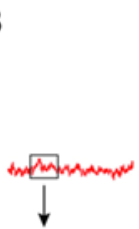

C

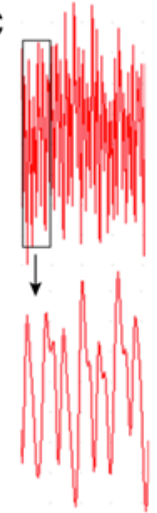

$\mathrm{D}$

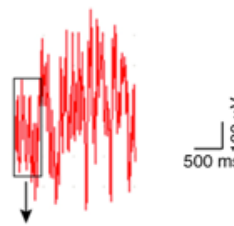

Control-EEG

Epilepsy-EEG1

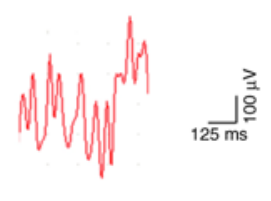

Epilepsy-EEG2

Figure 1. Changes in body weight and EEG following SE in rats. (A) Changes in body weight at each time point following SE. M1 represents the body weight of the rats before they were administered lithium chloride on the first day of the model; M2 represents the weight of the rat before they were administered pilocarpine on the second day of the model. (B) Local magnification of EEG in the control group. (C and D) Local magnification of EEG in the SE group. ${ }^{*} \mathrm{P}<0.05,{ }^{* * *} \mathrm{P}<0.001$. EEG, electroencephalogram; SE, status epilepticus.

value was higher in the SE group than in the control group $(\mathrm{P}<0.05, \mathrm{CA} 1$ area; $\mathrm{P}<0.01, \mathrm{CA} 3$ and $\mathrm{DG}$ areas). The numbers of EMAP II-positive cells peaked on day 3, and the optical density value of the SE group was significantly higher than that of the control group $(\mathrm{P}<0.001, \mathrm{CA} 1$ and CA3 areas; $\mathrm{P}<0.01, \mathrm{DG}$ area). The expression then decreased, although the numbers of EMAP II-positive cells were still significantly higher at 21 days in the SE group than in the control group (Figs. 3 and S2).

According to the results of western blot analysis, EMAP II expression was not altered significantly over time in the control group. However, the EMAP II protein expression levels were significantly higher in the SE group at the same time points vs. the control group. EMAP II protein expression began to gradually increase after the seizures began, peaking at 7 days $(\mathrm{P}<0.001)$ and then gradually declining. Nonetheless, the levels were still significantly higher at 21 days than those in the control group (Fig. 4).

Changes in the expression of ZO-1 and occludin in the hippocampal region of rats with $S E$. Western blot analysis revealed that, compared with the control group at the same time points, the protein expression of ZO-1 was significantly decreased at each time point in the SE group. ZO-1 protein expression was most evidently decreased at $2 \mathrm{~h}$ after SE and

then gradually increased until 14 days, at which point the expression of ZO-1 protein was still lower than that of the control group. Compared with the control group at the same time point, the protein expression of occludin was significantly decreased in the SE group at each time point. At $2 \mathrm{~h}$ following $\mathrm{SE}$, occludin protein expression was at its lowest level, but then gradually increased before decreasing again (Fig. 5).

\section{Discussion}

In the present study, an animal model of epilepsy was established via an intraperitoneal injection of lithium chlorine-pilocarpine, with EEG monitoring confirming that epileptic spikes were discharged in the experimental rats. Within $24 \mathrm{~h}$, the rats still had frequent convulsions, and a small number of rats died, with a $24-\mathrm{h}$ mortality rate of approximately $10.4 \%$, which was similar to that in a previous study (24). We also found that the weights of the rats had decreased significantly by $24 \mathrm{~h}$ before gradually recovering. After 7 days, the weights of the rats began to recover and they exhibited the same weight as the rats of the same age in the control group at 14 days after model establishment. As the durations of convulsions in the experimental group included in the present study all exceeded $30 \mathrm{~min}$, the current model can be used to study SE and may better reflect the degree of brain injury in rats.

Hypoxia and brain ischemic injury are always involved in the process of epilepsy $(21,27)$. Long-term seizures always cause brain hypoxia, and hypoxia can inflict a direct toxic injury on endothelial cells, as well as secondary blood-brain barrier (BBB) damage. Such secondary damage includes abnormal mitochondrial metabolism in endothelial cells, a thickening of the basal membrane, compensatory endothelial cell proliferation, and the presence of abnormal tight junctions $(28,29)$.

Angiogenesis and the destruction of the BBB are closely related to the occurrence and development of epilepsy. In the rat model of SE induced by pilocarpine, cerebral vessels exhibit varying degrees of morphology, with changes in, for example, distribution, density, length, angiogenesis, BBB opening, and cerebral blood flow and velocity (28). Recently, it was found that the expression level of occludin and ZO-1 in the neocortical microvessels of patients with drug-resistant temporal lobe epilepsy was significantly decreased compared with autopsy samples (30). In the present study, vascular endothelial cells were labeled through CD31 immunofluorescence and it was found that the numbers of vascular endothelial cells in each part of the rat hippocampus were significantly increased at $24 \mathrm{~h}$ following SE and that the numbers of vascular endothelial cells continued to be higher than that of the control group 21 days after SE. At the same time, it was also found that the expression levels of ZO-1 and occludin proteins in the SE group were significantly decreased at each time point, which is consistent with previous reports $(20,30,31)$. Tight junctions are important for the composition of the BBB. The increased vascular density following SE is accompanied by decreased expression of tight junction proteins, which indicates pathological angiogenesis. The downregulation of tight junction proteins further confirmed the increase in BBB permeability following SE, which would lead to the abnormal exchange of substances inside and outside blood vessels. This 


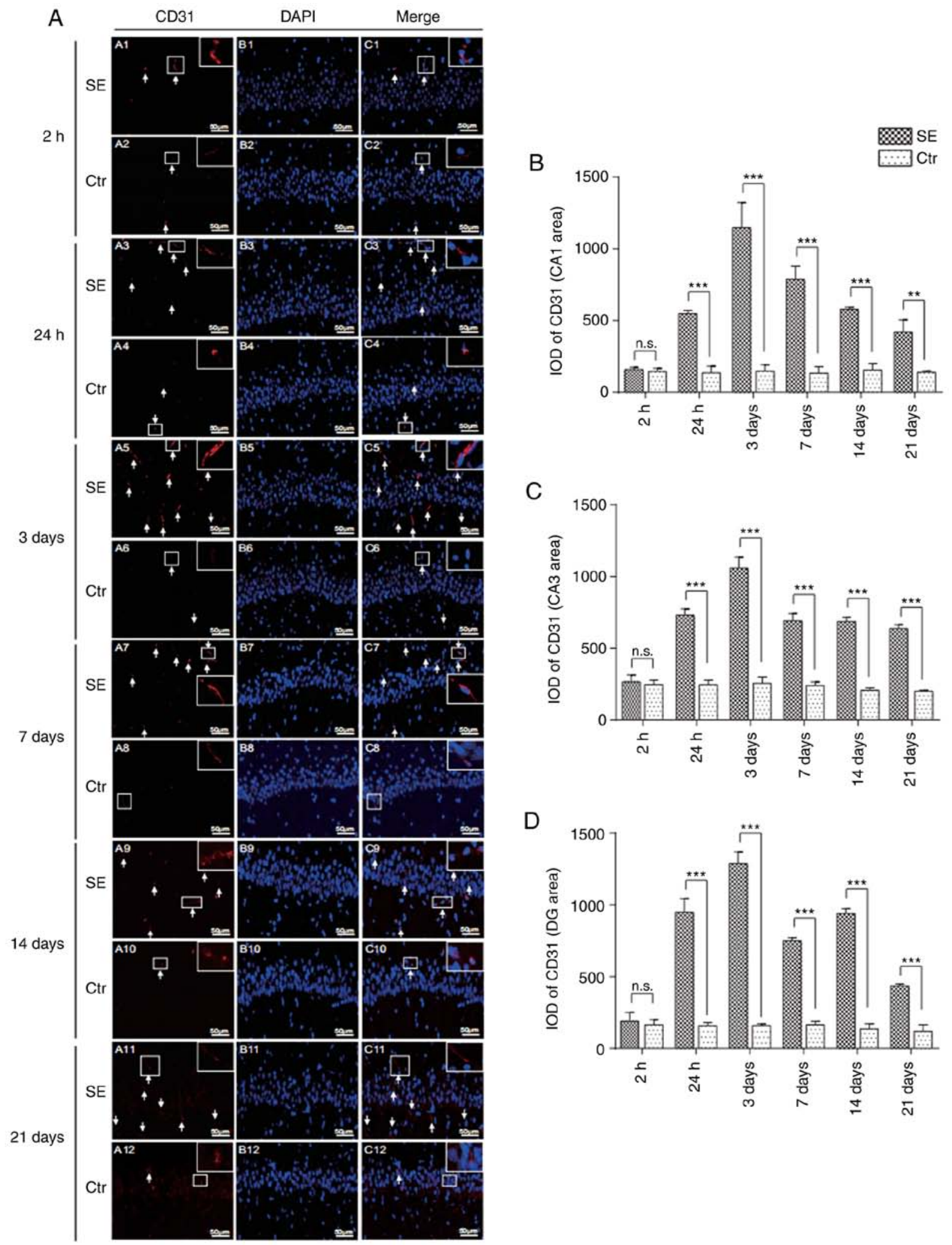

Figure 2. Changes in the numbers of CD31-positive endothelial cells in the hippocampus of rats with SE (x400 magnification). (A) Changes in numbers of CD31-positive endothelial cells in the CA1 region of the hippocampus. (A1-A12) CD31-positive endothelial cells labeled with Alexa Fluor 647 (red). (B1-B12) Nuclei labeled with DAPI (blue). (C1-C12) Co-localization of CD31-positive cells with DAPI. White arrows represent CD31-positive cells. (B-D) Density analysis of CD31 immunofluorescence in the CA1, CA3 and DG regions of the hippocampus at different time points. ${ }^{* * *} \mathrm{P}<0.01,{ }^{* * *} \mathrm{P}<0.001 ; \mathrm{n} . \mathrm{s}$., not significant. SE, status epilepticus.

would make the distribution of neurotransmitters in the brain abnormal and aggravate the occurrence and development of epilepsy (28).
EMAP II is a type of tissue factor that can induce endothelial cells to exert coagulant activity. A number of animal and clinical studies have found that EMAP II is 

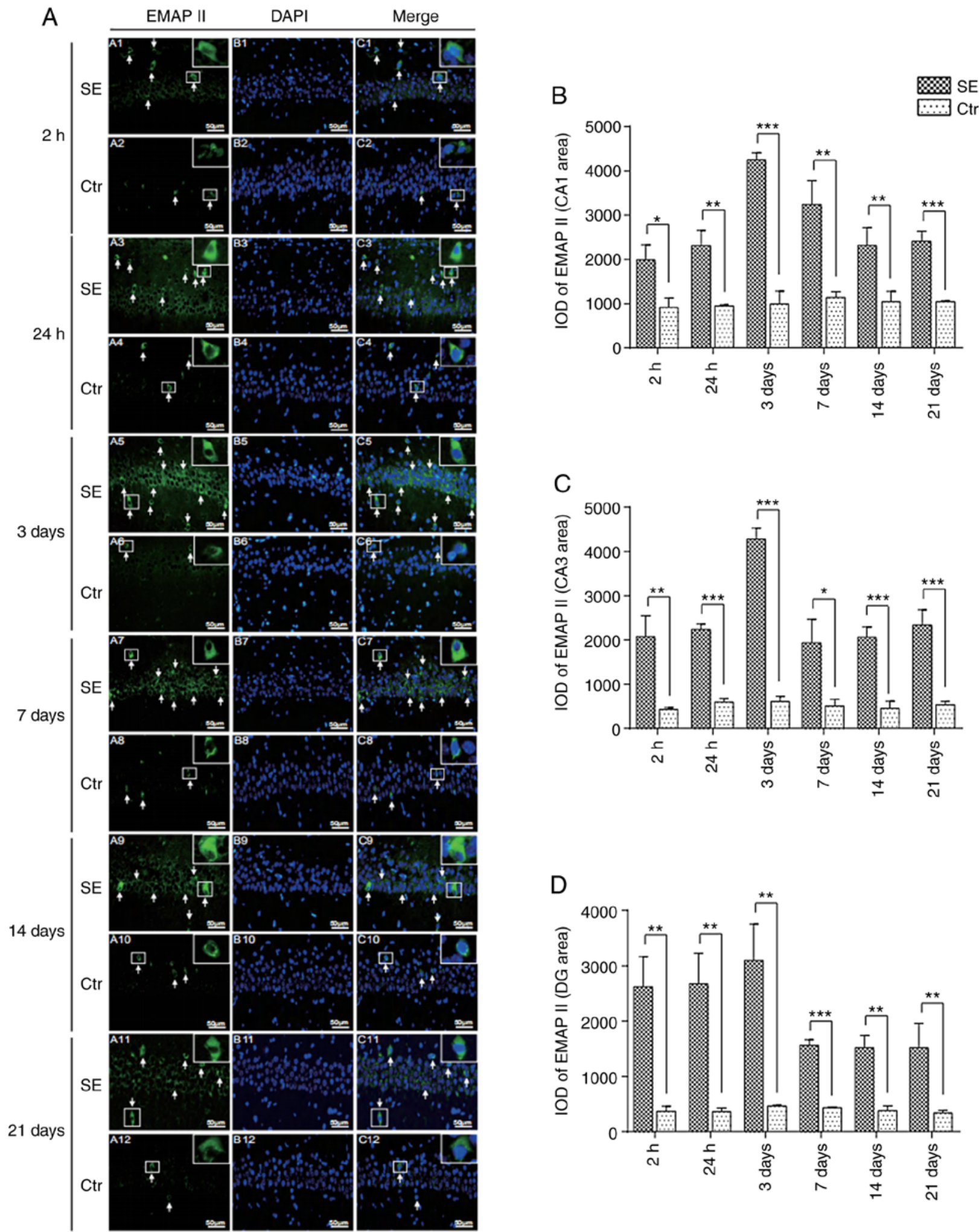

Figure 3. Changes in the numbers of EMAP II-positive cells in the hippocampus of rats with SE (x400 magnification). (A) Changes in numbers of EMAP II-positive cells in the CA1 region of the hippocampus. (A1-A12) EMAP II-positive cells labeled with Alexa Fluor 488 (green). (B1-B12) Nuclei labeled with DAPI (blue). (C1-C12) Co-localization of EMAP II with DAPI. White arrows represent EMAP II-positive cells. (B-D) Density analysis of EMAP II immunofluorescence in the CA1, CA3 and DG regions of the hippocampus at different time points. ${ }^{*} \mathrm{P}<0.05,{ }^{* *} \mathrm{P}<0.01,{ }^{* * *} \mathrm{P}<0.001$. EMAP II, endothelial monocyte-activating polypeptide II; SE, status epilepticus.

highly expressed in endocrine organs, particularly neuroendocrine organs (17,32). In addition, cerebral ischemia injury can induce EMAP II in brain tissue (33). Brabeck et al (34) found that the EMAP II-positive cell number was proportional to the severity of the brain damage induced by trimethyltin. Schikorski et al (12) found, through the analysis of nerve repair, that EMAP II expression was significantly higher within $24 \mathrm{~h}$, suggesting that EMAP II plays an important role 


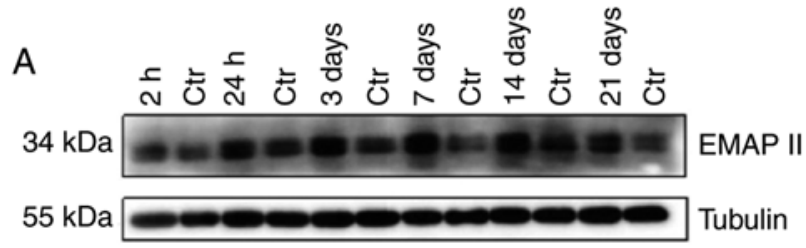

$\mathrm{B}$

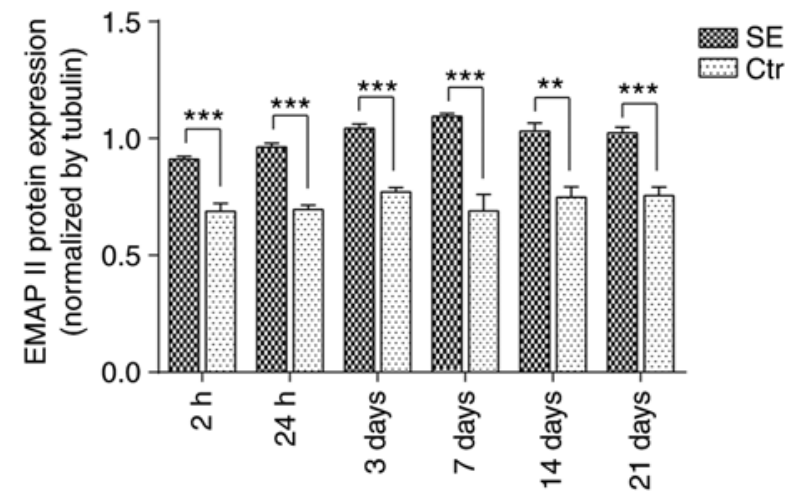

Figure 4. EMAP II protein expression in the hippocampus of rats with SE. (A) Changes in the expression of EMAP II and tubulin in the SE and control groups at the same time points. (B) Relative changes in the expression of EMAP II and tubulin in the SE and control groups at different time points. ${ }^{* *} \mathrm{P}<0.01,{ }^{* * *} \mathrm{P}<0.001$. EMAP II, endothelial monocyte-activating polypeptide II; SE, status epilepticus.

in the process of tissue regeneration. The present study found that the hippocampal EMAP II expression of the SE group began to significantly increase at $2 \mathrm{~h}$ after SE compared with the normal control group, continuing until 21 days. As reported in previous studies, epileptogenesis is related to hypoxia and apoptosis $(21,22)$, and the conversion of AIMP1 to EMAP II is enhanced under hypoxic conditions and apoptosis (15-17). The present study demonstrated that the expression of EMAP II increased after SE, suggesting that EMAP II maybe related to epileptogenesis.

In the present study, EMAP II expression was significantly increased at all time points following SE, while the expression of ZO-1 and occludin proteins decreased. It has been reported that a low expression of EMAP II could selectively increase blood-tumor barrier (BTB) permeability via a transcellular pathway through the RhoA/Rho kinase signaling pathway (35). EMAP II has also been reported to decrease the levels of tight junction-related proteins, ZO-1 and occluding, through the RhoA/ROCK and PKC signaling pathways in rat brain microvascular endothelial cells (BMECs) $(36,37)$. Based on these findings, it was hypothesized speculate that endogenously increased EMAP II may also decrease ZO-1 and occludin proteins to change the tight junction integrity of ECs following SE.

Park et al (38) found that the angiogenesis regulatory function of the EMAP II precursor protein AIMP1 was dependent on its concentration. At low concentrations, AIMP1 mediates angiogenesis, whereas, at high concentrations, AIMP1 leads to apoptosis of vascular endothelial cells and angiogenesis inhibition. Their results revealed that AIMP1 may inhibit angiogenesis through the C-terminal hydrolysate EMAP II. Clinically, owing to EMAP II inhibiting the growth of blood vessels, it is widely applied in the treatment of tumors at
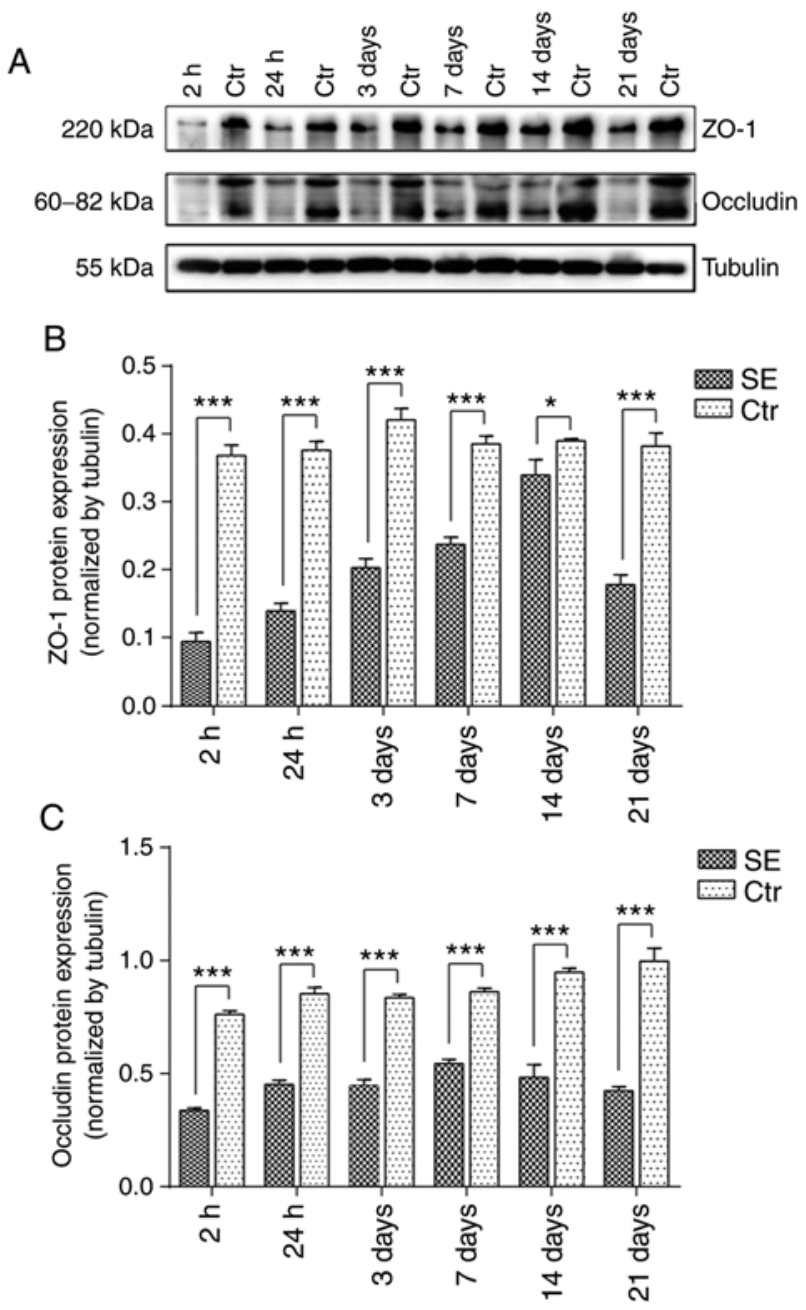

Figure 5. ZO-1 and occludin protein expression in the hippocampus of SE rats. (A) Changes in the expression of $\mathrm{ZO}-1$, occludin and tubulin in the $\mathrm{SE}$ and control groups at the same time points. (B) Relative changes in the expression of ZO-1 and tubulin in the SE and control groups at different time points. (C) Relative changes in the expression of occludin and tubulin in the SE and control groups at different time points. ${ }^{*} \mathrm{P}<0.05,{ }^{* * * *} \mathrm{P}<0.001$. EMAP II, endothelial monocyte-activating polypeptide II; SE, status epilepticus.

relatively high concentrations $(39,40)$. The present study demonstrated that the increased expression of EMAP II was accompanied by increased numbers of CD31-positive microvascular endothelial cells. It was hypothesized that endogenous EMAP II expression may be present at a relatively low concentration, which can promote angiogenesis following SE. Limitations of the present study include the absence of immunofluorescence analysis of ZO-1 and occludin, and the absence of detailed mechanism through which EMAP II alters angiogenesis and tight junction integrity following SE. Research is currently ongoing to explore the mechanisms exogenous EMAP II influences the progression of SE.

In conclusion, the expression of EMAP II in the rat hippocampus was found to be upregulated in the model of SE, which may promote angiogenesis with an increased number of CD31-positive microvascular endothelial cells. Moreover, an increase in endogenous EMAP II may also alter the tight junction integrity of BMECs, with a decreased expression of ZO-1 and occludin. However, further studies are required 
to reveal the exact mechanisms underlying the effects of EMAP II in the model of epilepsy.

\section{Acknowledgements}

Not applicable.

\section{Funding}

The present study was supported by the National Key Research and Development Program of China (grant no. 2016YFC1306203).

\section{Availability of data and materials}

The datasets used and/or analyzed during the current study are available from the corresponding author on reasonable request.

\section{Authors' contributions}

CL, WM and HW were involved in the conception and design of the study and the drafting of the manuscript. CL and WM were involved in the analysis and interpretation of the data. YZ assisted in setting up the protocols. CL and WM performed all the experiments and acquired all data. All authors read and approved the manuscript.

\section{Ethics approval and consent to participate}

The China Medical University Institutional Animal Care and Use Committee (No. 2016PS201K) approved all procedures.

\section{Patient consent for publication}

Not applicable.

\section{Competing interests}

The authors declare that they have no competing interests.

\section{References}

1. Thurman DJ, Begley CE, Carpio A, Helmers S, Hesdorffer DC, $\mathrm{Mu}$ J, Touré K, Parko KL and Newton CR: The primary prevention of epilepsy: A report of the prevention task force of the international league against epilepsy. Epilepsia 59: 905-914, 2018.

2. Fiest KM, Sauro KM, Wiebe S, Patten SB, Kwon CS, Dykeman J, Pringsheim T, Lorenzetti DL and Jetté N: Prevalence and incidence of epilepsy: A systematic review and meta-analysis of international studies. Neurology 88: 296-303, 2017.

3. Bertini G, Bramanti P, Constantin G, Pellitteri M, Radu BM, Radu M and Fabene PF: New players in the neurovascular unit Insights from experimental and clinical epilepsy. Neurochem Int 63: 652-659, 2013.

4. Takemiya $\mathrm{T}$ and Yamagata $\mathrm{K}$ : Intercellular signaling pathway among Endothelia, astrocytes and neurons in excitatory neuronal damage. Int J Mol Sci 14: 8345-8357, 2013.

5. Mogylnytska LA: Endothelial monocyte-activating polypeptide-II: Properties, functions, and pathogenetic significance. Fiziol Zh 61: 102-111, 2015 (In Ukrainian).

6. Kao J, Houck K, Fan Y, Haehnel I, Libutti SK, Kayton ML, Grikscheit T, Chabot J, Nowygrod R, Greenberg S, et al: Characterization of a novel tumor-derived cytokine. Endothelial-monocyte activating polypeptide II. J Biol Chem 269: 25106-25119, 1994
7. Yuan C, Yan L, Solanki P, Vatner SF, Vatner DE and Schwarz MA: Blockade of EMAP II protects cardiac function after chronic myocardial infarction by inducing angiogenesis. J Mol Cell Cardiol 79: 224-231, 2015.

8. Lal CV and Schwarz MA: Vascular mediators in chronic lung disease of infancy: Role of endothelial monocyte activating polypeptide II (EMAP II). Birth Defects Res A Clin Mol Teratol 100: 180-188, 2014.

9. Adly AAM, Ismail EA, Tawfik LM, Ebeid FSE and Hassan AAS: Endothelial monocyte activating polypeptide II in children and adolescents with type 1 diabetes mellitus: Relation to micro-vascular complications. Cytokine 76: 156-162, 2015.

10. Li Z, Liu XB, Liu YH, Xue YX, Wang P, Liu LB, Liu J, Yao YL and Ma J: Roles of serine/threonine phosphatases in low-dose endothelial monocyte-activating polypeptide-II-induced opening of blood-tumor barrier. J Mol Neurosci 57: 11-20, 2015.

11. Ma J, Meng F, Li S, Liu L, Zhao L, Liu Y, Hu Y, Li Z, Yao Y, $\mathrm{Xi} \mathrm{Z}$, et al: Autophagy induction by endothelial monocyte activating polypeptide II contributes to the inhibition of malignant biological behaviors by the combination of EMAP II with rapamycin in human glioblastoma. Front Mol Neurosci 8: 74, 2015.

12. Schikorski D, Cuvillier-Hot V, Boidin-Wichlacz C, Slomianny C, Salzet $M$ and Tasiemski A: Deciphering the immune function and regulation by a TLR of the cytokine EMAPII in the lesioned central nervous system using a leech model. J Immunol 183: 7119-7128, 2009.

13. Zhu X, Liu Y, Yin Y, Shao A, Zhang B, Kim S and Zhou J: MSC p43 required for axonal development in motor neurons. Proc Natl Acad Sci USA 106: 15944-15949, 2009.

14. Armstrong L, Biancheri R, Shyr C, Rossi A, Sinclair G, Ross CJ, Tarailo-Graovac M, Wasserman WW and van Karnebeek CD: AIMP1 deficiency presents as a cortical neurodegenerative disease with infantile onset. Neurogenetics 5: 157-159, 2014.

15. Knies UE, Behrensdorf HA, Mitchell CA, Deutsch U, Risau W, Drexler HC and Clauss M: Regulation of endothelial monocyte-activating polypeptide II release by apoptosis. Proc Natl Acad Sci USA 95: 12322-12327, 1998.

16. Barnett G, Jakobsen AM, Tas M, Rice K, Carmichael J and Murray JC: Prostate adenocarcinoma cells release the novel proinflammatory polypeptide EMAP-II in response to stress. Cancer Res 60: 2850-2857, 2000.

17. Murray JC, Barnett G, Tas M, Jakobsen A, Brown J, Powe D and Clelland C: Immunohistochemical analysis of endothelial-monocyte-activating polypeptide-II expression in vivo. Am J Pathol 157: 2045-2053, 2000.

18. Clauss M, Voswinckel R, Rajashekhar G, Sigua NL, Fehrenbach H, Rush NI, Schweitzer KS, Yildirim AÖ, Kamocki K, Fisher AJ, et al: Lung endothelial monocyte-activating protein 2 is a mediator of cigarette smoke-induced emphysema in mice. J Clin Invest 121: 2470-2479, 2011.

19. Rigau V, Morin M, Rousset MC, de Bock F, Lebrun A, Coubes P, Picot MC, Baldy-Moulinier M, Bockaert J, Crespel A, et al: Angiogenesis is associated with blood-brain barrier permeability in temporal lobe epilepsy. Brain 130: 1942-1956, 2007.

20. Morin-Brureau M, Lebrun A, Rousset MC, Fagni L, Bockaert J, de Bock F and Lerner-Natoli M: Epileptiform activity induces vascular remodeling and zonula occludens 1 downregulation in organotypic hippocampal cultures: Role of VEGF signaling pathways. J Neurosci 31: 10677-10688, 2011.

21. Ingram J, Zhang CF, Cressman JR, Hazra A, Wei Y, Koo YE, Žiburkus J, Kopelman R, Xu J and Schiff SJ: Oxygen and seizure dynamics: I. Experiments. J Neurophysiol 112: 205-212, 2014.

22. Wu Q and Wang $\mathrm{H}$ : The spatiotemporal expression changes of $\mathrm{CB} 2 \mathrm{R}$ in the hippocampus of rats following pilocarpine-induced status epilepticus. Epilepsy Res 148: 8-16, 2018.

23. Curia G, Longo D, Biagini G, Jones RS and Avoli M: The pilocarpine model of temporal lobe epilepsy. J Neurosci Methods 172: 143-157, 2008.

24. Glien M, Brandt C, Potschka H, Voigt H, Ebert U and Löscher W: Repeated low-dose treatment of rats with pilocarpine: Low mortality but high proportion of rats developing epilepsy. Epilepsy Res 46: 111-119,2001.

25. Racine RJ, Burnham WM and Gartner JG: First trial motor seizures triggered by amygdaloid stimulation in the rat. Electroencephalogr Clin Neurophysiol 35: 487-494, 1973.

26. Paxinos G and Watson C: The Rat Brain in Stereotaxic Coordinates - the New Coronal Set. 5th edition. Academic Press, New York, 2005. 
27. Li J, Jiang G, Chen Y, Chen L, Li Z and Wang Z and Wang X: Altered expression of hypoxia-Inducible factor-1 $\alpha$ participates in the epileptogenesis in animal models. Synapse 68: 402-409, 2014.

28. Ndode-Ekane XE, Hayward N, Gröhn O and Pitkänen A: Vascular changes in epilepsy: Functional consequences and association with network plasticity in pilocarpine-induced experimental epilepsy. Neuroscience 166: 312-332, 2010.

29. Friedman A and Heinemann U: Role of blood-brain barrier dysfunction in epileptogenesis. In: Jasper's Basic Mechanisms of the Epilepsies [Internet]. 4th edition. Noebels JL, Avoli M, Rogawski MA, Olsen RW and Delgado-Escueta AV (eds) National Center for Biotechnology Information (US), Bethesda, MD, USA, pp1-12, 2012.

30. Castañeda-Cabral JL, Colunga-Durán A, Ureña-Guerrero ME Beas-Zárate C, Nuñez-Lumbreras MLA, Orozco-Suárez S, Alonso-Vanegas M, Guevara-Guzmán R, Deli MA, Valle-Dorado MG, et al: Expression of VEGF- and tight junction-related proteins in the neocortical microvasculature of patients with drug-resistant temporal lobe epilepsy. Microvasc Res 132: 104059, 2020.

31. Kim JY, Ko AR, Hyun HW and Kang TC: ETB receptor-mediated MMP-9 activation induces vasogenic edema via $\mathrm{ZO}-1$ protein degradation following status epilepticus. Neuroscience 304: $355-367,2015$.

32. Li Z, Liu YH, Xue YX, Xie H and Liu LB: Role of ATP synthase alpha subunit in low-dose endothelial monocyte-activating polypeptide-II-induced opening of the blood-tumor barrier. J Neurol Sci 300: 52-58, 2011.

33. Liao YL, Zhang ZY, Liu JW, Schluesener HJ, Zhang ZR and $\mathrm{Wu}$ YZ: Lesional expression of EMAPII in macrophages/microglia following cerebral ischemia in rats. Int $\mathrm{J}$ Neurosci 121: 58-64, 2011.
34. Brabeck C, Michetti F, Geloso MC, Corvino V, Goezalan F, Meyermann R and Schluesener HJ: Expression of EMAP-II by activated monocytes/microglial cells in different regions of the rat hippocampus after trimethyltin-induced brain damage. Exp Neuro 177: 241-246, 2002.

35. Li Z, Liu YH, Liu XB, Xue YX, Wang P and Liu LB: Low-dose endothelial monocyte-activating polypeptide-II increases permeability of blood-tumor barrier via a PKC- $\zeta / \mathrm{PP} 2 \mathrm{~A}$-dependent signaling mechanism. Exp Cell Res 331: 257-266, 2015.

36. Li Z, Liu YH, Xue YX, Liu LB and Xie H: Mechanisms for endothelial monocyte-activating polypeptide-II-induced opening of the blood-tumor barrier. J Mol Neurosci 47: 408-417, 2012.

37. Xie H, Xue YX, Liu LB, Liu YH and Wang P: Role of RhoA/ROCK signaling in endothelial-monocyte-activating polypeptide II opening of the blood-tumor barrier: Role of RhoA/ROCK signaling in EMAP II opening of the BTB. J Mol Neurosci 46: 666-676, 2012.

38. Park SG, Kang YS, Ahn YH, Lee SH, Kim KR, Kim KW, Koh GY, Ko YG and Kim S: Dose-dependent biphasic activity of tRNA synthetase-associating factor, p43, in angiogenesis. J Biol Chem 277: 45243-45248, 2002.

39. Schwarz RE, Awasthi N, Konduri S, Caldwell L, Cafasso D and Schwarz MA: Antitumor effects of EMAP II against pancreatic cancer through inhibition of fibronectin-dependent proliferation. Cancer Biol Ther 9: 632-639, 2010.

40. Awasthi N, Schwarz MA, Verma V, Cappiello C and Schwarz RE: Endothelial monocyte activating polypeptide II interferes with VEGF-induced proangiogenic signaling. Lab Invest 89: 38-46, 2009. 